\title{
Comparison of Chinese and American Health Care System
}

\author{
Weining Wang
}

University of Delaware, Newark, DE, 19716, US

\begin{abstract}
Health care is an indispensable part of people's lives, and public health is the foundation of a nation's prosperity. The United States is one of the most advanced countries in the world, and China is developing rapidly. However, the health care systems in both countries are different. Each system has its advantages and disadvantages, so by learning from each other's strengths, the two systems can be complementary.

Keywords: Health care system; Patients and hospitals; Improvement and suggestions;
\end{abstract}

\section{Introduction}

\subsection{Research background and significance}

In recent years, with the rapid development of globalization, China's economy has entered a rapidly developing stage, and the subsequent regional development imbalance has become more obvious [1]. In particular, attention needs to be paid to the domestic imbalance in medical security [2]. As medical and health services are closely related to people's health and interests, China's medical system has become a focus of social concern. China has the largest population in the world, and the improvement of people's living standards has put forward higher requirements for the national medical and health security. Although our country has made great progress in various aspects of medical treatment through the reform of medical system and mechanism, many problems remain to be solved due to a lack of experience [3].

The United States is the world's largest spender on health care. After decades of development and improvement, it is undeniable that the United States has had the most mature health care system in the world. Therefore, the health care system of the United States has become the focus of debate and attention of governments and scholars of various countries around the world. This paper aims to put forward ideas for mutual learning in the reform and practice of the two countries by comparing the medical security systems of China and the United States under different national conditions.

\subsection{Research directions and methods}

This study mainly compares the similarities and differences between Chinese and American medical systems (including medical security implementation and supervision system, medical forms and so on), medical resources (equipment, training level, etc.), medical technology, hospital outpatient volume, medical expenses, and hospitals' scientific research level (satisfaction degree of research-oriented hospitals and patients). The main research methods involved are literature investigation and comparative analysis. The research data came from the official websites of the OECD, the World Health Organization (WHO), the World Bank, the U.S. Centers for Disease Control, as well as "the Statistical Yearbook of Health and Family Planning in China". The rigorous literature investigation and comparative analysis results can help not only explain the differences and advantages of China and the United States in the medical security system under the development of the times and the changes of national conditions, but also provide a practical basis for the reasonable improvement of relevant institutions. Meanwhile, the ideas and results of the paper also play a role in assisting in the work and study of relevant researchers.

\section{Investigation Directions and Results}

\subsection{Differences in health care systems between China and the United States}

First of all, since the 1980s, China has begun the reform of the medical care system, thus forming the current urban medical security system that includes social medical insurance, public medical care, urban medical assistance system and other forms coexist [4]. However, although the most common community health care has been paid much attention to by the country, it has not been fully included in the basic medical and health services. Secondly, the implementation of national essential drugs promotes the scientific and rational drug use [5]. However, 90\% of Chinese hospitals are public ones, and implementing the market mechanism $\mathrm{n}$ public medical institutions to pursue services and generate income has greatly reduced the fairness of medical care. Thirdly, the mechanism in line with hospital assessment isn't perfect, which leads to the 
conflicts between doctors and patients.

Unlike China, firstly, America is the only country that doesn't offer universal health insurance among the developed countries in the world. Medical insurance is divided into private medical insurance and social medical insurance [6]. Secondly, public hospitals account for only about $20 \%$ of the total number of hospitals in the United States, and private hospitals occupy a larger share. Doctors work in partnership with hospitals, and they only use their medical facilities to practice medicine. Thirdly, the U.S. public health system includes national, state and local health administration and research institutions, as well as rigorous disease monitoring systems.

\subsection{Medical resources and medical technologies}

Hospitals in China are classified into three levels, and they are further divided into township level, county level, prefectural level, provincial level and ministerial level [7]. Hospitals with different grades represent different levels of medical care. High-grade hospitals have relatively complete equipment, the doctors' technology is more mature. When Chinese hospitals introduce advanced treatment technologies and methods, they need to pass a rather complicated approval mechanism, which delays their application.

In the United States, hospitals are classified into medical centers and general hospitals, so as to ensure the relative fairness of medical resources. It is known to all that the United States has the world's advanced medical equipment and technology. Lung cancer, breast cancer and prostate cancer have the highest death rate in China and the United States. Taking the cancer with the highest occurrence rate for example, there is an very obvious gap in the medical level between China and the United States. The national cancer center of China released the latest statistical results in January 2019. The results indicated that the incidence rate of malignant tumors in 2015 was about 3.929 million, with an increase of 125,000 and $3.2 \%$ compared with 3.804 million in 2014, and one person dies of cancer every 5 minutes. In the United States, there are 392 cases of cancer per 100,000 people, and 163.5 people die of cancer.

The relatively advanced medical technology in the United States is closely related to its medical system, scientific research technology, doctors' training and remuneration.

\subsection{The number of outpatients in the hospital}

In China, due to the large population and the shortage of medical resources, the number of outpatients in Chinese hospitals is far greater than that in American hospitals. Taking children's hospital as an example, American children's hospitals have an annual outpatient volume of about 100,000, while Shanghai children's hospital has an annual outpatient volume of 2 million [8]. In addition, the number of people working in Chinese children's hospitals is far greater than that in American children's hospitals, which also reflects the shortage of resources in today's China.

\subsection{Clinic costs}

In the United States, advanced equipment and resources lead to high medical costs, while in China, prices are relatively acceptable. America's non-universal health insurance results in a high threshold for many commercial insurance claims, while China's government-controlled health care system makes reimbursement have a low threshold.

\subsection{Scientific research ability}

As for the training of doctors, China pays more attention to results-based practice, so the focus of doctors in the internship is always on learning clinical skills. Although current hospitals limit the promotion of doctors by the scientific research level, this forces busy doctors to choose academic fraud.

America has rigorous admission standards and more uniform medical education. Teaching hospitals in the United States are mostly large advanced hospitals. Meanwhile, interns and doctors must complete teaching and scientific research tasks. In addition, the United States ranks first in terms of scientific research means, equipment and speed.

\subsection{Patients'satisfaction degree}

In China, patients are faced with the pressure of material and physical health, while doctors are faced with both great work stress and mental stress, so they may easily conflict with each other. Due to the lack of insurance and laws in this respect, once disputes occur, patients can only let hospitals solve the problem, and the conflict between doctors and patients escalates. Therefore, patients will only become more dissatisfied with the reality of difficult and expensive medical services

However, in the United States, doctors generally have their own medical liability insurance established by their lawyers and hospitals, and they also have sound treaties to deal with medical disputes. Once medical treatment disputes occur, doctor and patient need not meet, and the hospital lawyer and patient lawyer will negotiate and settle the claim according to the agreement, which greatly reduces unnecessary troubles. 


\section{Discussion and Conclusion}

\subsection{Results analysis and discussion}

As shown in Table 1, by the end of 2014, the number of beds per 1,000 people in China was much higher than that in the United States, while the number of doctors per 1,000 people was lower than that in the United States. As shown in Table 2, during 1980-2012, the number of hospital admissions in the United States showed a low-speed decline, while the number of outpatients increased year by year [9]. In China, both hospital admissions and outpatients presented a rising trend. The results in Figure 1 show that the average daily hospitalization in China and the United States decreases year by year, but the average hospitalization days in China are still much higher than those in the United States. As shown in Figure 2, the utilization rate of hospital beds is also decreasing year by year in the United States, while in China, it is much higher than that in the United States [10].
Table 1. number of beds per 1,000 people The number of beds The number of doctors per 1000 people per 1000 people

\begin{tabular}{|c|c|c|c|c|}
\multicolumn{1}{c}{ per 1000 people } & \multicolumn{1}{c|}{ per 1000 people } \\
\hline Year & U.S & China & U.S & China \\
\hline 1960 & 9.18 & $\ldots$ & 1.10 & $\ldots$ \\
\hline 1970 & 7.88 & 7.88 & 1.20 & 0.86 \\
\hline 1980 & 6.01 & 6.01 & 1.50 & 1.18 \\
\hline 1990 & 4.86 & 4.86 & 1.80 & 1.56 \\
\hline 2000 & 3.49 & 3.49 & 2.56 & 1.68 \\
\hline 2014 & 2.93 & 2.93 & 2.56 & 2.12 \\
\hline
\end{tabular}

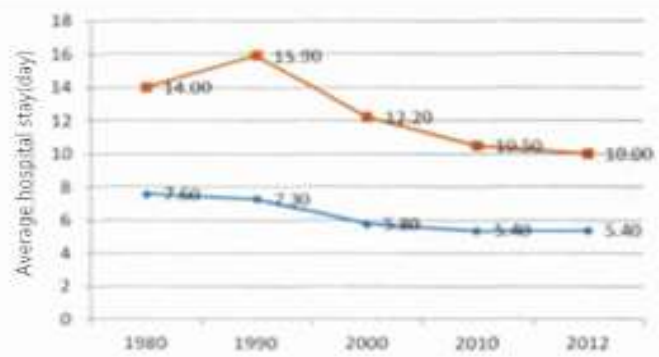

Figure 1. average daily hospitalization
Table 2. Number of hospital admission
U.S
China

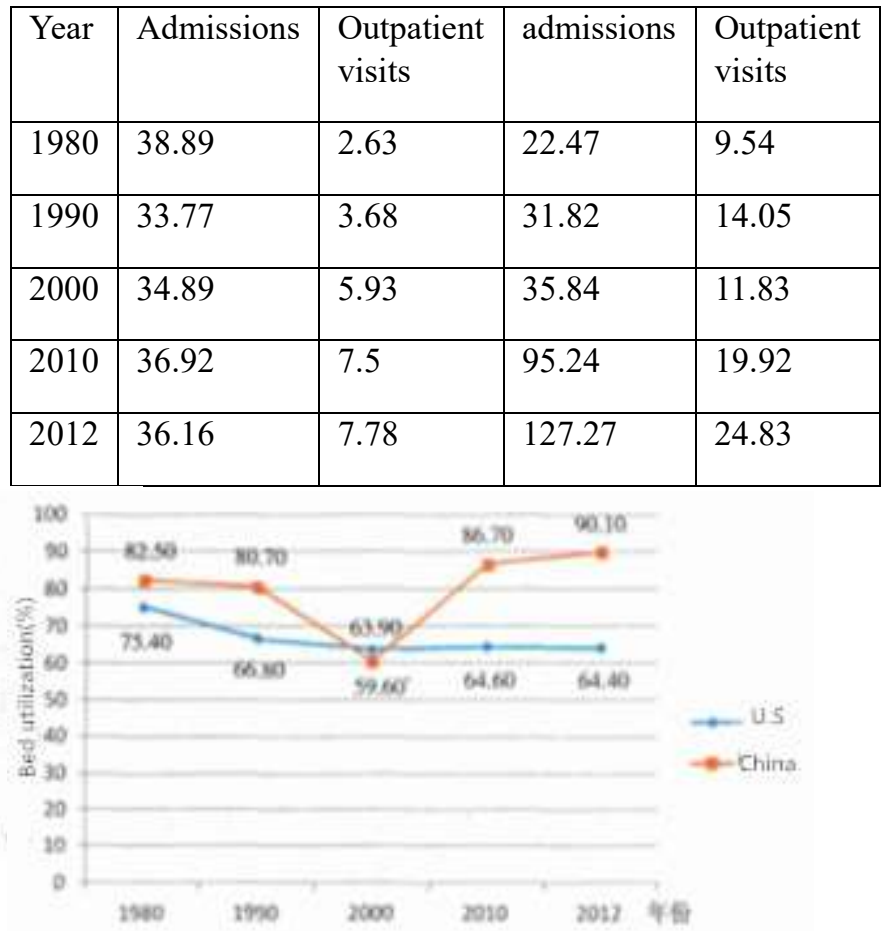

Figure 2. utilization rate of hospital beds

Notes: Statistical Yearbook of Chinese Health and Family Planning in 2015; Outpatient and admission visits in the United States came from the statistical data of all hospitals, including federal and non-federal hospitals, community hospitals, mental hospitals, tuberculosis control hospitals, and other specialized hospitals. The number of outpatients and admission visits in China came the statistical data of hospitals, excluding primary medical and health institutions, specialized hospitals and nursing homes.

\subsection{Conclusions}

On the whole, in the last century, China's medical level and resources were far behind the United States, but in the past three decades, China's development has narrowed the gap between the two countries. There are two main reasons for this. First of all, economic development has led to people's increasing demand for medical care as well as sense of health and wellness. Secondly, China and the United States are faced with different health conditions, so they have different responses to sanitary conditions.

The ration of medical treatment and public health costs spent by the United States has always been ranking top one in the world, so the government is more willing to control the rising medical costs and pay attention to medical costs. They control the rising costs by limiting health service 
items. Therefore, America's health policy aims to pay for health care by restructuring the supply of services. But for China, addressing the shortage of health resources and inadequate system is the key to medical reform. The increasing variety of medical methods and medical insurance systems largely reduce the problem of difficult access to medical care. However, many abuses of resources still hide behind the huge amount of medical treatment, so that many people cannot feel the effects of health reform.

\section{Improvement and Suggestions on the Medical System of Both Sides}

\subsection{China}

First of all, due to the limited and uneven distribution of health resources in China as well as the unreasonable structure configuration, community services and rural health undertakings are the focus of the next construction, so as to form the trend of wide coverage. At the same time, increasing the input and introduction of resources can fundamentally solve the problem. Secondly, because the medical security system isn't perfect, people spend a lot on treating diseases, so expanding the coverage of basic health care and reducing the out-of-pocket rate can be a good way to address the medical problems of the poor. Thirdly, compared with the United States, the implementation of China's hospital management mechanism still has some shortcomings. Implementing effective monitoring and management can help improve the price of medicines and medical services and make them more transparent. Fourthly, learning from the diversified experience of the United States in running hospitals and forming a healthy competition in the medical service industry can make relevant staff more active, which can not only provide patients with the right to choose from a variety of options and reduce doctor-patient conflicts, but also improve the efficiency of the medical service industry.

\subsection{The United States}

Currently, the major problems faced by the U.S. health care industry are high costs and low coverage. First of all, family health care system can provide remote residents with telemedicine services. Secondly, special monitoring departments are needed to control the price of medical devices as well as doctors' treatment. Thirdly, the amount of medical insurance and subsidies for the disadvantaged and the poor shall be enhanced, and the overpriced insurance products shall be appropriately suppressed.

\section{REFERENCES}

[1] Yang Yongheng, Hu Angang, Zhang Ning, Regional Disparity and Incoordination of Human Development in China --- "One China, four worlds" from a historical perspective. Economics (quarterly), 2006. 5(3): p. 803816.

[2] Deng feng, Lv Juhong, Gao Jianmin, et al. Comparative Analysis of Medical Resources and Health Costs in Brics Countries. China health economics, 2014. 33(2): p.94-96.

[3] Wang Dehua, Zhang Qiong, Public Medical Insurance and Residents' Medical Burden. Research on China's Economic Transformation and Development, 2008.

[4] Sun Yanming, Reflection on Current Health Resource Allocation and Government Responsibility. East China Economic Management, 2006. 6: p. 62-67.

[5] Meng Qingyue, Cheng Gang, Sun Xiaojie, Study on Effects of Medicine Price Policy on Medicine Cost Control. China Health Economics, 2004. 4: p. 50-52.

[6] Song Yuan, Ben Ying. Compensation Methods of Foreign Medical Services and Their Enlightenment to China. China Health Eesources, 2011, 4.

[7] Statistical Information Center of the Ministry of Health, China Health Service Survey: The Third National Health Service Survey Analysis Report. Beijing: Chinese Peking Union Medical College, 2004.

[8] Jiang Tianwen, Fan Zhihong, Behavior Distortion Mechanism and Process Analysis of Chinese Medical System. Economic Research, 2002. 11: p. 71-80.

[9] World Bank. World Development. Indicators[EB/OL]. [2016-03-06]. http://data.worldbank.org/.

[10] SCHIMIDT R, G.S., SPRECKELSEN C., Decision support for hospital bed management using adaptable individual length of stay estimations and shared resources $\mathrm{BMC}$ med inform decis mak, 2013. 13(3). 